Klein, W. (2005). Vom Sprachvermögen zum Sprachsystem. Zeitschrift für

Literaturwissenschaft und Linguistik, 140, 8-39.

Wolfgang Klein

Vom Sprachvermögen zum sprachlichen System

Schon als Tier hat der Mensch Sprache.

Johann Gottfried Herder

\title{
1. Einleitung
}

Herders berühmte Abhandlung über den Ursprung der Sprache, 1769 als Antwort auf eine Preisaufgabe der Preussischen Akademie der Wissenschaften verfaßt, zu lesen ist mühselig. Der Stil ist nach heutigen Maßstäben pathetisch, und die Argumentation ist weithin von Fakten frei, eine Eigenschaft, die sie mit den meisten der gut und gern 15000 - 20000 bekannten Abhandlungen über den Ursprung der Sprache teilt ${ }^{1}$. Aber der erste Satz, der hier als Motto zitiert wird, drückt in sieben Worten zwei Vorstellungen aus, mit deren einer sein Verfasser fast allen Zeitgenossen - nicht zuletzt den Naturwissenschaftlern des 18. Jahrhunderts - voraus war und deren andere klarsichtiger ist als manches, was heute zu über den Ursprung der Sprache zu lesen ist. Mit ersterer meine ich die Vorstellung, daß sich der Mensch aus einem Tier entwickelt hat und in gewisser Weise auch noch ein Tier ist. Herder war ein hoher Geistlicher, Mitglied des Oberkonsistoriums im Herzogtum Weimar, und nach allem was wir wissen, ein frommer Christ; aber die Idee der Evolution war ihm selbstverständlich. Das Zitat ist auch kein Ausrutscher. In seinem ein gutes Jahrzehnt später verfaßten Hauptwerk 'Ideen zu eine Philosophie der Geschichte der Menschheit" heißt es:

Mancherlei Verbindungen des Wassers, der Luft, des Lichts mußten vorhergegangen sein, ehe der Same der ersten Pflanzenorganisation, etwa das Moos, hervorgehen konnte. Viele Pflanzen mußten hervorgegangen und gestorben sein, ehe eine Tierorganisation ward; auch bei dieser gingen Insekten, Vögel, Wasser- und Nachttiere den gebildetern Tieren der Erde und des Tages vor, bis endlich nach allen die Krone der Organisation unsrer Erde, der Mensch, auftrat. (Herder 1784, S. 34)

Diesen Gedanken führt er auf gut 150 Seiten im Detail aus. Herder hatte natürlich keine Vorstellung von der Funktionsweise der Evolution, so wie sie uns die moderne Genetik vermittelt. Aber beschreibt die damals bekannten evolutionären Fakten bis hin zum Übergang vom Affen zum Menschen. Drei Eigenschaften sind es, die uns vom Orang Utan - den er als den engsten Bruder des Menschen ansieht - abheben. Das sind erstens physiologische Eigenschaften, insbesondere der aufrechte Gang und eine "feinere Organisation der Nerven" (nicht übrigens die Größe des Gehirns), zweitens eine gewisse moralische Unterlegenheit des Menschen ("Kein Tier mordet sein Geschlecht auf Befehl eines dritten mit kaltem Blut", S. 173), und drittens schließlich Sprache und Kognition, die zur Ausbildung der höheren Kultur geführt haben.

\footnotetext{
${ }^{1}$ Hewes (1975) führt 11000 Titel an, und seither sind mindestens 5000 hinzugekommen.
} 
Ebendie Verbindung von Sprache und Kognition ist es auch, die das Wesen der menschlichen Sprache ausmacht.

Die zweite wichtige Vorstellung nämlich, die in dem einleitenden Zitat zum Ausdruck kommt, ist die, dass Sprache nicht Sprache ist. Tiere haben die Möglichkeit, ihre Emotionen unmittelbar auszudrücken und sich mit anderen ihrer Art zu verständigen. In diesem Sinne hat auch der Mensch als Tier Sprache:

Alle Tiere, bis auf den stummen Fisch, tönen ihre Empfindung: deswegen aber hat doch kein Tier, selbst nicht das vollkommenste, den geringsten, eigentlichen Anfang zu einer menschlichen Sprache. Man bilde und verfeinere und organisiere dies Geschrei, wie man wolle; wenn kein Verstand dazukommt, diesen Ton mit Absicht zu brauchen, so sehe ich nicht, wie nach dem vorigen Naturgesetze je menschliche, willkürliche Sprache werde. Kinder sprechen Schälle der Empfindung, wie die Tiere; ist aber die Sprache, die sie von Menschen lernen, nicht ganz eine andre Sprache?

Aber was die menschliche Sprache ausmacht, ist etwas ganz anderes. Es ist die Fähigkeit, unabhängig von konkreten emotionalen Zuständen und unabhängig von kommunikativen Zwecken Vorstellungen mit Lautgebilden zu verknüpfen. Sein Beispiel ist das eines blökenden Schafes, für das der Mensch sich eine naheliegende Lautfolge wählt, die sich fest und unabhängig von dieser Situation mit der Vorstellung eines solchen Schafes verknüpft. Nur der Mensch hat dieses Vermögen, nur der Mensch macht Gebrauch davon, und zwar ganz unabhängig davon, ob er die so im Gedächnis verankerte Verbindung von Laut und Bedeutung dann zu kommunikativen Zwecken verwendet.

Ich erwähne all dies hier nicht als eine kleine Verbeugung vor einer großen, wenn auch etwas obskuren und selten gelesenen Gestalt der deutschen Geistesgeschichte, sondern weil Herders Ausführungen in einigen Punkten weniger einfältig ist als ein nicht geringer Teil der heutigen Debatte über die biologische Seite des Menschen im allgemeinen und der Sprache im besonderen. Er hat nicht den geringsten Zweifel, daß die Evolution des Menschen aus elementarsten organischen Formen mit seinen religiösen Überzeugungen vereinbar ist. Der Glaubenskrieg zwischen Evolutionstheorie und Kreationismus wäre ihm lächerlich vorgekommen. Die Sprache ist durch eine biologische Entwicklung zustandegekommen; ${ }^{2}$ es ist auffällig, wie scharf er sich gegen die Vorstellung einer Gottesgabe wendet. aber es ist sinnlos, einfach von "Sprache" zu reden. Sprache ist vieles, und die Sprache des Menschen, soweit sie nicht das ist, was er schon als Tier hat, ist die speziesspezifische Fähigkeit, Ausdruckssysteme zu entwickeln, in denen Lautfolgen und Bedeutungen verknüpft sind. Dies wiederum ist die Voraussetzung jeder höheren Kultur, wie sie nun einmal den Menschen auszeichnet und wie er sie im Laufe seiner Geschichte entwickelt hat.

Damit soll es aber auch genug des Lobes sein. Herder redet nie von der Syntax, sondern von einzelnen Wörtern, und auch da sind seine Ausführungen zumeist sehr nebulös. So ist das, was er über die Entstehung der ersten Wörter sagt, ungefähr so nebulös wie das, was man heute darüber sagt. Damit sind wir bei der Frage angelangt, um die es im folgenden geht:

Wie kommt man vom biologisch gegebenen, speziesspezifischen Sprachvermögen zu sprachlichen Systemen, und welche Rolle spielt die Kommunikation darin?

Dieser Frage wird in den folgenden vier Abschnitte nachgegangen.

${ }^{2}$ Bemerkenswert übrigens, wie scharf er sich an vielen Stellen gegen die Vorstellung wendet, die Sprache sei ein Geschenk Gottes. 


\section{Sprachvermögen - sprachliche Systeme - Kommuniktion}

Seit Saussure, spätestens seit Saussure, ist es üblich, zwischen drei Begriffen von Sprache zu unterscheiden:

- das jedem Menschen angeborene Sprachvermögen ("faculté de language")

- das einzelsprachliche System, das im Lauf der Geschichte entwickelt wird ("langue")

- der tatsächliche Gebrauch, der von einem solchen System gemacht wird ("parole").

Für Saussure und für die gesamte strukturelle Linguistik steht der mittlere Begriff, der des einzelsprachlichen Systems, im Zentrum der sprachwissenschaftlichen Forschung: "La linguistique a pour unique et véritable objet la langue envisagée en elle-même et pour elle-même" heißt der berühmte Schlußsatz des "Cours de linguistique générale". In der Linguistik, wie Saussure sie versteht, geht es um die strukturellen Eigenschaften des Deutschen, des Chinesischen oder des Tagalog und um die Entwicklung dieser Eigenschaften im Laufe der Jahrhunderte. Um diese Eigenschaften zu erforschen, muß man den Sprachgebrauch studieren, und insofern ist die "parole" auch Gegenstand der Untersuchung. Der Zusammenhang zwischen "langue" und "parole" ist oft und kontrovers diskutiert werden, sowohl in der Tradition des klassischen Strukturalismus wie in der Tradition der generativen Grammatik, dort unter dem Stichwort Kompetenz vs Performanz. Erstere bezieht sich auf das Wissen des Sprechers von "seiner Sprache", letztere auf den tatsächlichen Gebrauch, den er davon macht. Anders als die Saussuresche langue, die überindividuell - ein "fait social" - ist, ist die Kompetenz an das Individuum gebunden; aber in einer gängigen und sinnvollen Idealisierung wird nicht das Wissen des realen Sprechers betrachtet, sondern daß eines idealen, der "seine Sprache" perfekt kennt, und damit verwischt sich der Unterschied. In der generativen Tradition spielt, anders als in der des klassischen Strukturalismus, auch der dritte Begriff, der des Sprachvermögens ("language faculty"), eine wesentliche Rolle. Das hängt mit der Vorstellung zusammen, die Sprache sei in erster Linie als ein "mentales Organ" aufzufassen, das sich unter dem Einfluß von Umgebungsfaktoren etwas unterschiedlich ausprägt; diese Ausprägungen sind die einzelnen sprachlichen Systeme. Von wissenschaftlichem Interesse sind nicht so sehr diese Ausprägungen mit all ihren Besonderheiten, sondern das, was ihnen gemeinsam ist - und dies wiederum sind die Eigenschaften des mentalen Organs Sprache. Ich werde gleich darauf zurückkommen, gehe jetzt aber zunächst etwas näher auf das "Sprachvermögen" ein.

\section{3. $\mathrm{K} 1, \mathrm{~K} 2, \mathrm{~K} 3$}

Das Sprachvermögen ist allen Menschen mehr oder minder gemeinsam; einzelsprachliche Systeme - ich werden im folgenden oft kurz von "Ausdruckssystemen" reden - gibt es derzeit vielleicht 5000 oder 6000, und in der Geschichte der Menschheit muß es sehr viel mehr gegeben haben. Wiekommt man von ersterem zu letzteren? Hier gibt es prinzipiell zwei Möglichkeiten, nämlich Sprachschöpfung und Spracherwerb.

Jedes normale Kind lernt im Verlaufe eines guten Jahrzehnts eine Sprache, seine Muttersprache. Die meisten Menschen lernen eine Reihe weiterer Sprachen, vielleicht nicht bis zur Perfektion - wie immer man "perfekt" in diesem Zusammenhang definieren mag -, aber doch bis zu einem gewissen Grad der Beherrschung. Was das Kind, oder allgemeiner gesprochen, der Lerner zu leisten hat, ist, BESTIMMTE REGULARITÄTEN EINES SYSTEMS ZU KOPIEREN. Er muß lernen, daß die Lautfolge / kniga/ Buch bedeutet, daß der Akkusativ davon gebildet wird, indem man /a/ durch / u/ ersetzt, daß ein bestimmtes Verb den Akkusativ regiert, 
daß die finite Komponente des Verbalkomplexes im Nebensatz am Ende steht, usw. usw. - es gibt tausende und abertausende solcher sprachspezifischer Eigenschaften, und um eine Sprache wie Russisch, Deutsch oder Chinesisch zu lernen, muß der Lerner diese Eigenschaften aus dem Input kopieren. Dazu setzt ihn das angeborene Sprachvermögen imstande. Dieses "Kopiervermögen" ist weitestgehend speziesspezifisch, auch wenn sich gewisse Ansätze bei anderen Arten finden.

Die Fähigkeit, eine Sprache zu lernen, muß irgendwann im Verlauf der Evolution entstanden sein. Wir wissen nicht, wann dies war. Wir wissen aber, daß es damals noch keine sprachlichen Systeme gegeben hat. Das Sprachvermögen kann daher nicht nur darin bestehen, die Regularitäten einer gegebenen Sprache, sei es in der Lexik oder der Grammatik, zu kopieren, sondern es muß auch eine konstruktive Komponente umfassen - ebenjene Komponente, die es unsern fernen Ahnen ermöglicht hat, erstmals ein solches System zu entwickeln. Dabei ist offen, ob dies nur einmal geschehen ist und sich alle Sprachen aus dieser einen entwickelt haben, oder ob es die Sprachschöpfung ein gängiger Prozeß war, der sich in verschiedenen Weltgegenden immer wieder ereignet hat. Eines ist jedoch klar. Das biologisch gegebene Sprachvermögen hat funktional gesehen zwei ganz verschiedene Komponenten - nämlich das "Konstruktionsvermögen”, d.h. die Fähigkeit, ein Ausdruckssystem zu schaffen, und das schon genannte Kopiervermögen, d.h. die Fähigkeit, die Besonderheiten eines bestehenden Systems zu reproduzieren. Erstere erlaubt die Sprachschöpfung, letzteres den Spracherwerb; dabei ist jedoch die jeweils andere Komponente mitbeteiligt.

Es muß zumindest eine weitere funktionale Komponente im Sprachvermögen geben nämlich die Fähigkeit, ein Ausdruckssystem zu kommunikativen Zwecken zu nutzen. Diese Fähigkeit existiert auch, wenn kein Gebrauch von ihr gemacht wird. Jemand kann viele Sprachen kennen und in vielen Sprachen schweigen. Die Fähigkeit zu kommunizieren, setzt die Kenntnis eines Ausdrucksystems voraus, geht aber weit darüber hinaus. Sie verlangt beispielsweise, daß dieses Ausdruckssysstem gezielt mit zahlreichen anderen Fähigkeiten unseres Geistes in Bezug gesetzt wird. Etwas anders gesagt, die Fähigkeit zu sprechen und zu verstehen, ist NICHT das Wissen, das ein Sprecher von seiner Sprache hat; es ist die Fähigkeit, dieses Wissen zu nutzen.

Wir müssen also mindestens drei Komponenten des Sprachvermögens klar unterscheiden das Konstruktionsvermögen, das Kopiervermögen und das Kommunikationsvermögen. (ich werde diese drei Komponenten im folgenden gelegentlich als K1, K2 und K3 bezeichnen). Diese Unterschiedung bezieht sich auf bestimmte Leistungen des Sprachvermögens; sie ist funktional. Es ist eine offene Frage, wie diese drei Komponenten "hardwareseitig" zueinander stehen, d.h. inwieweit sie von denselben Teilen des Nervensystems und der peripheren Organe getragen werden. Letztere sind offenkundig bei allen drei Komponenten beteiligt. Bei ersteren ist dies weniger klar. Es wäre sicher absurd anzunehmen, daß es K1, K2 und K3 drei anatomisch klar abgrenzbare Module im Gehirn entsprächen. Auf der anderen Seite sind die drei Aufgaben aber auch vieles gemeinsam, sodaß die durchaus auch auf einer etwas unterschiedlichen Interaktion derselben Teile des Hirns beruhen. Wie immer - dies ist eine Frage der physiologischen Grundlagen des Sprachvermögens, nicht der funktionalen Eigenschaften des Sprachvermögens selbst, eine Frage für den Hirnforscher, nicht für den Linguisten.

Für den Linguisten interessant ist die Frage, wie die drei Komponenten des Sprachvermögens funktional zueinander stehen. Ein Kind, das Sorbisch lernt, muß alle strukturellen Besonderheiten des Sorbischen aus dem Input kopieren. Dies schließt aber nicht aus, daß neben dem Kopiervermögen auch das Konstruktionsvermögen eine Rolle in diesem Prozeß spielt. Strukturelle Besonderheiten aus dem Input zu kopieren, heißt ja nicht, eine Lautfolge zu imitieren, wie ein Tonbandgerät, sondern aus dem Input auf gewisse Regelhaftigkeiten zu schließen, die diesem Input zugrundeliegen. Hierfür mag es gewisse grundsätzliche Prinzipien geben, die bei der freien Konstruktion eines Ausdruckssystems zum Tragen kommen, die die Schöpfung der ersten Sprache oder der ersten Sprachen geleitet haben und die nach wie vor wirksam sein können. Diese Prinzipien müssen sich aber NICHT in der 
Struktur aller existierenden Sprachen wiederfinden, weil deren historische Entwicklung zu ganz anderen Systemen geführt haben kann; dieser Punkt wird in den folgenden Überlegungen eine wichtige Rolle spielen. Das Konstruktionsvermögen ist ja, wie das Kopiervermögen, zunächst einmal eine individuelle Fähigkeit. Der Austausch mit anderen kann aber zur Ausbildung von Strukturen führen, zu denen der einzelne entweder allein gar nicht fähig wäre oder - dies ist die plausiblere Annahme - die er normalerweise von sich nicht schafft, obwohl er es im Prinzip könnte.

Dieser Austausch ist durch K3 vermittelt - das Vermögen, Ausdruckssysteme zu kommunikativen Zwecken zu verwenden. Hier gerät man sowohl bei der Sprachschöpfung wie beim Spracherwerb in eine paradoxe anmutende Situation. Um eine Sprache schaffen zu können, muß man bereits kommunizieren können; um kommunizieren zu können, braucht man aber eine Sprache: K1 setzt K3 voraus, und umgekehrt. Wir wissen nicht, wie die erste Sprache - oder die ersten Spachen - entstanden sind. Aber es war sicher nicht so, daß sie sich jemand ausgedacht und dann seiner Familie und seinen nächsten Freunden mitgeteilt hat; wie denn? Die erste Sprache hat sich in der Kommunikation entwickelt. Entsprechendes gilt, allerdings mit einer charakteristischen Asymmetrie, für den Spracherwerb: der Lerner muß die Äußerungen, aus denen er lernt, in gewisser Weise verstehen und in der Regel auch produzieren; dazu muß er sie aber gelernt haben. Die Asymmetrie liegt darin, daß hier einer der Beteiligten in der Regel dieses Problem nicht hat - nämlich derjenige, aus dessen Input man die Regeln der jeweils zu lernenden Sprache extrapoliert. Für den Lerner gilt jedoch, daß K2 K3 voraussetzt, und wiederum umgekehrt. In beiden Fällen ist es jedoch kein wirkliches Paradox, und dies aus zwei Gründen. Erstens sind Sprachschöpfung wie Spracherwerb langfristige Prozesse: es werden bestimmte Teilsysteme ausgebildet, die in gewissen Grenzen eine Kommunikation erlauben; diese wiederum erlaubt die Ausbildung weiterer Teilsysteme. Zweitens können andere kommunikative Prozesse als die über ein sprachliches System zu Hilfe kommen.

Wenn es nun so ist, daß sowohl K1 wie K2 ein funktionierendes K3 verlangen, dann stellt sich sofort die Frage, inwieweit das Ergebnis - nämlich das resultierende Sprachsystem, sei es nun kopiert oder frei geschaffen - in seinen strukturellen Eigenschaften die Besonderheiten der Kommunikation widerspiegeln. Man beachte, daß es hier nicht um die Frage geht, inwieweit das menschliche Sprachvermögen sich - eventuell unter selektivem Druck - aus den Bedürfnissen der Kommunikation entwickelt hat ("Das Bedürfnis schafft sich sein Organ", Friedrich Engels, 1876). Dies mag der Fall sein oder auch nicht. Vielmehr geht es darum, ob die Ergebnisse, die dieses Vermögen zustande bringt, eben die Sprachsysteme, gewisse strukturelle Eigenschaften haben, die sich aus der Interaktion von K1, K2 und K3 ergeben.

Es sei noch einmal an die Ausgangsfrage erinnert: Wie kommt man vom biologisch gegebenen, speziesspezifischen Sprachvermögen zu sprachlichen Systemen, und welche Rolle spielt die Kommunikation darin? Offenbar ist das Bild ein höchst verwickeltes. Das Sprachvermögen hat zumindest drei funktionale Komponenten, und Ausdruckssysteme entstehen aus der Interaktion dieser Komponenten; daher werden auch die strukturellen Eigenschaften dieser Systeme von diesen drei Komponenten diktiert. Was sind diese Eigenschaften? Bevor wir uns dieser Frage zuwenden, will ich kurz auf eine - die derzeit bekannteste - Vorstellung vom Zusammenhang zwischen Sprachvermögen und Sprachsystem eingehen, jene, die in etwas variierender Form seit einem halben Jahrhundert der generativen Grammatik zugrundeliegt.

\section{Ein einfaches Bild}

There is a language faculty. Chomsky, wiederholt 
Fragen der Sprachschöpfung wie auch Fragen einer Universalgrammatik waren in der ernsthaften Sprachwissenschaft, sowohl, sowohl der traditionellen wie der strukturellen, lange verpönt; die Societé der Linguistique von Paris hat sie in ihrer Satzung von 1866 sogar ausdrücklich verboten; in Artikel 2 heißt es lakonisch: "La Société n'admet aucune communication concernant, soit l'origine du langage, soit la création d'une langue universelle." Dieses Verdikt hat sich für ein gutes Jahrhundert auch außerhalb dieser gelehrten Gesellschaft gehalten. Kaum ein Sprachwissenschaftlicher von Rang hat sich zum Ursprung der Sprache äußern mögen. Das hat sich seit gut dreißig Jahren geändert; seither gibt es eine Fülle von Tagungen, Sammelbänden, Aufsätzen und Monographien zur Frage des Sprachursprungs (siehe z.B. Ward und Trabant 2001, Wunderlich 2002, Tallerman 2005). Dennoch ist es nach wie vor für die meisten Linguisten ein exotisches Thema, mehr ein Gegenstand teils wilder, teils subtiler Spekulation und wissenschaftlich ernstzunehmen nur für einige Randbereiche, wie die Entwicklung der peripheren Organe.

Durchaus kein exotisches Thema ist die zweite Art des Übergangs vom Sprachvermögen zum sprachlichen System - der Spracherwerb. Es gibt seit gut einem Jahrhundert eine intensive Forschung, die zunächst vorrangig von Psychologen betrieben wurde. Seit Jakobsons berühmten Werk über Kindersprache, Aphasie und allgemeine Lautgesetze von 1941 haben sich zunehmend auch Linguisten unterschiedlicher Couleur damit befaßt. Dieser Aufschwung hängt eng mit dem hohen Stellenwert zusammen, der dem Spracherwerb - genauer gesagt, der Möglichkeit des Spracherwerbs - in der generativen Grammatik zukommt. Jedes normale Kind, so eine für die weitere Entwicklung der generativen Grammatik prägende Annahme Chomskys bereits in den Fünfzigerjahren, lernt seine Muttersprache im Verlauf einiger weniger Jahre bis zur Perfektion, und zwar schnell, mühelos und - dies der wichtigste Punkt - auch bei fehlerbehaftetem und unzulänglichem Input seitens der Eltern und all der anderen, mit denen das Kind kommuniziert. Insbesondere beherrscht das Kind auch grammatische Strukturen, die sich im Input, der ihm bis zu einem gegebenen Zeitpunkt zugänglich war, selten oder gar nicht finden. Dies ist nicht durch induktive Prozesse des Lernens zu erklären, wie sie beispielsweise in den damals herrschenden behavioristischen Lerntheorien angenommen wurden und auch heute meist noch angenommen werden. Vielmehr muss ein wesentlicher Teil der Sprachbeherrschung angeboren sein und lediglich in den ersten Lebensjahren aktiviert werden. Da aber keinem eine bestimmte Sprache angeboren ist, ist der Kern der menschlichen Sprachfähigkeit universal allen Menschen und allen Sprachen gemeinsam. Der eigentliche Gegenstand der linguistischen Theorie ist daher die UNIVERSALGRAMMATIK.

Dieser Gedanke hat sich durch alle Wandlungen der generativen Grammatik in den letzten fünfzig Jahren erhalten. Er ist allerdings durch verschiedene Überlegungen ergänzt und in gewissen Grenzen auch modifiziert worden. Diese Abwandlungen beziehen sich sowohl auf die zugrundeliegende Philosophie wie auf die empirische Ausgestaltung. $\mathrm{Zu}$ ersterem zählt insbesondere die Vorstellung, daß die menschliche Sprache ein "mentales Organ" ist, nicht anders als physiologische Organe wie die Leber oder der Magen (Anderson and Lightfoot 2002). Dieses Organ hat sich zu irgendeinem Zeitpunkt der Menschwerdung durch genetische Veränderungen im Gehirn gebildet, und zwar ganz unabhängig von irgendwelchen funktionalen Aufgaben, die es erfüllen kann, insbesondere von der Kommunikation. Es ist klar, daß "Sprache" in diesem Sinne sich nicht auf einzelne sprachliche Systeme beziehen kann, denn die sind sicher nicht durch einmalige genetische Prozesse entstanden. Das mentale Organ "Sprache" ist ein innerer Zustand, der in den ersten Lebensjahren gewissen Veränderungen unterworfen ist; bei diesen Veränderungen spielen Umweltfaktoren, und hier insbesondere der sprachliche Input aus der sozialen Umgebung, eine gewisse, freilich sehr beschränkte Rolle. Der Anfangszustand genauer gesagt, unsere Vorstellung vom Anfangszustand - ist die UNIVERSALGRAMMATIK, d.h. jene Form des Sprachvermögens, das sich noch nicht unter dem Einfluß des Inputs zu einer 
Grammatik mit sehr spezifischen Eigenschaften ausgebildet hat. Der Endzustand, in der Regel um die Pubertät erreicht, ist die Beherrschung einer bestimmten Sprache. Der Spracherwerb ${ }^{3}$ ist daher eine begrenzte Umweltanpassung eines von Natur aus gegebenen, in Grenzen entwicklungsfähigen Organs. In Chomskys eigenen Worten:

'the language organ is the faculty of language (FL); the theory of the initial state of FL, an expression of the genes, is universal grammar (UG); theories of states attained are particular grammars; the states themselves are internal languages, "languages" for short', (Chomsky 2002, 64)

Eine interne Sprache ist nicht das Ausdruckssystem selbst, sondern das Wissen eines Sprechers, in dem ein solches Ausdruckssystem repräsentiert ist. In dieser Vorstellung wird angenommen, daß nur solche interne Repräsentationen einen sinnvollen Gegenstand wissenschaftlicher Untersuchungen bilden können. "Externe Sprachen", so wie sie seit den Tagen der griechischen Grammatiker im Mittelpunkt aller sprachwissenschaftlichen Bemühungen stehen, sind schlecht definierte Objekte und nur am Rande von wissenschaftlichem Interesse.

Es ist schwer zu sagen, ob die Rede vom mentalen Organ, seinem Anfangszustand und seinem Endzustand, mehr als eine faģon de parler ist, der, weil sie keine substantiellen Annahmen macht, eigentlich jeder zustimmen kann. Ein Kind, das Deutsch lernt, muß halt lernen, daß die Lautfolge /bu:x/ "Buch" bedeutet, daß der Genitiv /bu:xs/ lautet, daß helfen den Dativ regiert, unterstützen hingegen den Akkusativ, und daß das finite Verb im Hauptsatz an zweiter Stelle steht, ganz egal, welche Konstituente vorausgeht. Dies kann man als Veränderung eines inneren Organs unter dem Einfluß von Umweltfaktoren bezeichnen; aber das ist keine sehr aussagekräftige Redeweise. Dazu müßte Genaueres über die Prozesse der Veränderung und die ihnen zugrundeliegenden Regularitäten gesagt werden; ebenso müßte spezifiziert werden, wie der Anfangszustand beschaffen ist, was also die Eigenschaften der Universalgrammatik sind.

Dazu gibt es in der generativen Tradition verschiedene Vorschläge, deren am sorgsamsten ausgearbeiteter das Modell der "Principles and Parameters" ist, das zu Beginn der Achzigerjahre in die Diskussion eingebracht wurde (Chomsky 1981; die sorgfältigste Darstellung ist nach wie vor von Stechow und Sternefeld 1986). Eine leitende Vorstellung, von Chomsky selbst wiederholt als der bedeutende Fortschritt der Sprachwissenschaft in den letzten zwei Jahrtausenden apostrophiert, war die Idee, daß die Struktur von Sprachen nicht durch spezifische Regeln zu beschreiben ist, sondern durch allgemeine Prinzipien, die in ihrer Gesamtheit die Universalgrammatik ausmachen. Hier gibt es jedoch wohldefinierte Spielräume, die eine gewisse Variation erlauben; die Universalgrammatik gibt nicht alle Eigenschaften vor, nicht einmal alle wichtigen, sondern sie ist "parametrisiert".

Die gesamte Kompetenz eines Sprechers, der seine Sprache perfekt beherrscht, den Endzustand also erreicht hat, besteht aus einem "Kern" (core) und der "Peripherie". Letzteres sind alle idiosynkratischen Erscheinungen einer Sprache, also beispielsweise die besondere deutsche Form der Nominalflexion oder die besondere Laut-Bedeutung-Zuordnung eines einzelnen deutschen Wortes. Daß, um das obige Beispiel noch einmal aufzunehmen, das Buch im Deutschen /bu:x/ heisst und nicht beispielsweise /schu:/, dass der Genitiv davon /bu:xes/ ist und nicht /bo:x/ - dies sind Idiosynkrasien des Deutschen, zählt zur Peripherie. Der Kern ist Teil unserer genetischen Ausstattung, also angeboren, und er ist universal, da es keinen Grund

${ }^{3}$ Gemeint ist hier immer der Erstspracherwerb des Kindes. Es gibt viele Gründe für die Annahme, daß im Zweitspracherwerb Erwachsener das Kopiervermögen nachläßt, während das Konstruktionsvermögen durchaus erhalten bleibt. Deshalb können Erwachsene zwar sehr wohl weitere Sprachen lernen. Sie machen es nur nicht genauso, wie jene, von denen sie lernen. 
für die Annahme gibt, das es in dieser Hinsicht einen nennenswerten Unterschied in der genetischen Ausstattung gibt. Diese universale Komponente gibt die Struktur aller möglichen Entfaltungen weithin vor: sie legt in allen zentralen Punkten die Struktur der Einzelsprachen fest. Diese Struktur und die sie bestimmenden Prinzipien wurde im Rahmen des "P\&P-Ansatzes" mit viel Fleiß, Scharfsinn und Akkuratesse zu einer Reihe von Teiltheorien ausgearbeitet, die jeweils einen bestimmten Bereich empirischer Phänomene abdecken - X-bar-Theorie, Bindungstheorie, Kasustheorie u.a. Das Ergebnis dieser Mühen war, bei einer Reihe von divergenten Auffassungen im einzelnen, ein beeindruckendes Theoriengebäude, das eine Reihe von klaren Aussagen über die Beschaffenheit der Universalgrammatik und ihre Ausformung zu einzelnen Sprachen macht.

Diese Sprachen unterscheiden sich. Ein Teil der Unterschiede ist "peripher" im Sinne der Theorie. Jedoch läßt auch schon die Universalgrammatik läßt von Anfang an bestimmte Alternativen offen, die dann unter dem Einfluß der Umwelt eine unterschiedliche Ausprägung erfahren können. Ebendiese offenen Stellen sind die Parameter. Ein besonders einfaches Beispiel ist der sogenannte "Kopfparameter". Jede Konstruktion, so die Annahme, hat einen "Kopf" und ein "Komplement". In dem Ausdruck altes Eisen ist Eisen der Kopf und altes das Komplement. In dem Ausdruck zum alten Eisen ist $q u(m)$ der Kopf und (dem) alten Eisen das Komplement. In zum alten Eisen legen ist legen der Kopf und zum alten Eisen das Komplement. Die Universalgrammatik, also der Anfangszustand des Sprachorgans, läßt zunächst offen, ob das Komplement dem Kopf folgt oder im vorausgeht: diese Eigenschaft ist parametrisiert, sie muss einzelsprachlich unterschiedlich belegt werden. Die Attraktivität dieses Gedankens liegt nun darin, dass ein einzelner Parameter oft eine ganze Reihe von strukturellen Eigenschaften bündelt. Ein besonders oft diskutiertes Beispiel - das demnach auch im Spracherwerb am meisten untersucht wurde - ist der "Pro-drop parameter". Manche Sprachen, wie Deutsch, Englisch, Französisch, haben ein obligatorisches Subjektpronomen, während andere, wie Lateinisch, Italienisch, Spanisch, es auslassen können. Diese Eigenschaft des "pro-drop" korreliert nun mit einer Reihe weiterer, insbesondere einer relativ reichen Verbalmorphologie, einer vergleichsweise freien Wortstellung sowie mit der Möglichkeit, Elemente aus bestimmten Nebensätzen herauszubewegen (dem sogenannten "that-trace effect"). Der Pro-drop parameter besagt nun, dass all diese Eigenschaften davon abhängen, wie ein bestimmtes abstraktes Element der Satzstruktur ("AGR") parametrisiert ist. Man beachte, dass die Auslaßbarkeit des Subjektpronomens nicht der Parameter ist, ein Punkt, der in der Spracherwerbsforschung oft durcheinander gebracht wird; diese Auslaßbarkeit ist eine von verschiedenen strukturellen Konsequenzen einer bestimmten Parametrisierung. Wenn der Parameter also erst einmal gesetzt ist - und das kann der Lerner im Prinzip anhand jeder seiner strukturellen Konsequenzen im Input lernen -, dann folgen daraus automatisch auch alle anderen strukturellen Eigenschaften, darunter auch solche, die dem Input vielleicht nur sehr schwer zu entnehmen sind. Dies ist ein sehr eleganter Gedanke, der zumindest in einer Reihe von Fällen erklären könnte, wie sich der Lerner mit den vielen Idiosynkrasien zurecht findet, durch die sich Sprachen voneinander unterscheiden.

Der Übergang von Sprachvermögen zum sprachlichen System - oder, in der hier verwandten Redeweise, vom Anfangszustand zum Endzustand des Sprachorgans - hat demnach zwei Komponenten: alle Eigenschaften der Peripherie müssen aus dem Input gelernt werden, und ebenso muß die jeweils gültige Parametrisierung aus dem Input abgeleitet werden. Das wirft zwei Fragen auf: Erstens, welche Eigenschaften zählen zur Peripherie, welche zum Kern, und zweitens, was innerhalb des Kerns ist in welcher Weise parametrisiert? Die Antwort auf beide Fragen nimmt der Theorie viel von ihrem theoretischen Glanz. Aus dem Input gelernt werden muss auf jeden Fall der gesamte Wortschatz, ebenso die spezifische Form der verschiedenen morphologischen Teilsysteme, etwa die Nominalflexion, imgleichen das spezifische Phoneminventar, und nicht zuletzt alle Idiosynkrasien der einzelsprachlichen Syntax - mit anderen Worten, fast alles. Nicht gelernt werden muß, was universal ist, abgesehen von jenen 
Eigenschaften, in denen die Universalgrammatik parametrisiert ist. Was aber sind nun die einzelnen Parameter? Darüber gibt es in der generativen Grammatik keinerlei Einigkeit; die beiden obengenannten, Kopfparameter und pro-drop-Parameter, haben in der Erwerbsforschung eine grosse Rolle gespielt. Aber sie haben sich zum einen empirisch nicht bestätigen lassen, und sie sind inzwischen auch in der linguistischen Theorie selbst weithin aufgegeben. Dies macht es, bei aller Attraktivität des Gedankens, sehr schwer, seinen empirischen Gehalt zu überprüfen.

In der derzeit von Chomsky selbst vertretenen Variante der generativen Grammatik - von ihm selbst vorsichtig als "minimalistisches Programm" bezeichnet - sind die meisten bisherigen Vorstellungen über die Universalgrammatik aufgegeben, so etwa die der X-bar-Theorie, die die Basis der Syntax bildet. Aufgegeben ist auch die Idee des Parameters als Schüsselbegriff der Theorie. Es verbleiben lediglich einige sehr allgemeine Prinzipien der Strukturbildung, aus denen sich sehr wenig über den Weg vom Sprachvermögen zu sprachlichen Systemen herleiten läßt, eigentlich gar nichts (vgl. Chomsky 1995, 2002, 2005, und speziell zur Relevanz des minimalistischen Programms für den Spracherwerb Longa und Lorenzo, im Druck).

\section{Ausdrucksysteme}

\subsection{Verschieden}

Was zeichnet die Produkte des menschlichen Sprachvermögens vor anderen Instrumenten der Kommunikation aus? Zunächst einmal, daß sie erheblich variieren. Diese Variabilität betrifft praktisch alle Aspekte des sprachlichen Wissens, über das ein Sprecher verfügen muß, wenn er Latein, Chinesisch oder Kikscht können will:

- Lexikon: es gibt, abgesehen vielleicht von Coca Cola, keine lexikalische Einheit, die in allen Sprachen der Welt vorkäme.

-Syntax: manche Sprachen stellen das finite Verb regelhaft ans Ende des Satzes, manche zwischen Subjekt und Objekt, wieder andere an den Anfang; bei vielen variiert es mit dem Satztyp. Manche Sprachen markieren den Argumentstatus durch die Stellung, andere nicht. Im Englischen steht das direkte Objekt normalerweise nach dem Nomen, im Deutschen kann es davor, aber auch danach stehen, im Französischen steht es danach, wenn es sich um eine lexikalische Nominalphrase handelt, und davor, wenn es sich um ein Personalpronomen handelt - allerdings wiederum danach, wenn dieses Personalpronomen betont ist, usw. usw.

- Morphologie: Deutsch, Latein und Russisch haben, obwohl genetisch eng verwandt, kein einziges Flexionsmorphem gemeinsam; andere Sprachen, wie Chinesisch, haben überhaupt keine Flexionsmorphologie:

- Phonologie: zwar bewegt sich das Repertoire an Lauten, die der Mensch mit seinen peripheren Organen produzieren kann und unterscheiden kann, innert gewisser Grenzen, und die akustischen Eigenschaften, die zum Aufbau phonologischer Systeme genutzt werden, sind nach Zahl und Art erheblich beschränkt, aber was die einzelnen Systeme aus diesem biologisch vorgegebenen Repertoire machen, ist ganz verschieden.

- Semantik: alle Sprachen sind in der Lage, zeitliche und räumliche Relationen in differenzierter Weise auszudrücken; das Vorgehen im einzelnen unterscheidet jedoch erheblich. In den indogermanischen Sprachen hat jeder finite Satz unweigerlich eine 
Zeitmarkierung, gleich ob der Sprecher dies für wünschenswert hält oder nicht; andere Sprachen haben wiederum gar keine Tempusmarkierung am Verb. Manche Sprachen kodieren räumliche Relationen relativ zur Position des Sprechers, etwa in Ausdrücken wie bier, links, vorn, d.h., sie nutzen die Asymmetrien des menschlichen Körpers zur sprachlichen Strukturierung des Raumes. Andere nutzen keine solche Deiktika, oder jedenfalls nicht zu diesem Zweck.

\subsection{Gleich: die Trivia}

All dies schließt nicht aus, dass es in jedem dieser Bereiche auch universale Eigenschaften gibt; aber wenn dies der Fall ist, dann sind sie entweder trivial oder sehr abstrakt. Im folgenden will ich zunächst auf einige Eigenschaften aller sprachlichen Systeme eingehen, über die sich alle Sprachwissenschaftler mehr oder minder einig sind; dies sind sozusagen die Trivia, die K1 auf jeden Fall zu schaffen und K2 auf jeden Fall zu kopieren in der Lage sein muß. Dann werden einige Kandidaten für darüber einige nichttriviale Kandidaten für Universalia erörtert.

Die beiden Fundamentalannahmen, über die es von Aristoteles bis heute kaum einen Dissens gibt, sind:

(a) Sprachsysteme bestehen aus Ausdrücken, wobei ein Ausdruck ist eine Verbindung von "Bedeutung" und "Bedeutungsträger" ist. Die Terminologie schwankt, aber mit "Bedeutung" ist immer eine relativ abstrakte Einheit unserer Kognition gemeint, die sich sekundär auf bestimmte Elemente der Außenwelt bezieht, mit Bedeutungsträger im gängigsten Fall eine Lautfolge; es kann aber auch eine Folge von Schriftzeichen, von Handbewegungen oder dergleichen sein.

(b) Es gibt elementare Ausdrücke ("Wörter, Lexeme, lexikalische Einheiten") und komplexe Ausdrücke ("Phrasen, Sätze, Texte"); letztere werden nach bestimmten Regeln aus einfacheren Einheiten gebildet. Eine jede Sprache hat ein LEXIKON - das ist das Repertoire der elementaren Einheiten - und eine GRAMMATIK: das ist die Gesamtheit der Regeln (oder Prinzipien), nach denen sich komplexe Ausdrücke aus einfacheren bilden lassen. So haben es schon die alten Griechen gesagt, so gilt es auch noch heute.

K1, das Konstruktionsvermögen, besteht darin, solche Ausdrücke bilden zu können, und zwar in praktisch beliebig großer Zahl. Eine Kultursprache hat in aller Regel einige hunderttausend Wörter, und es gibt keine prinzipielle Grenze für die Menge elementarer Ausdrücke. Die Zahl der zusammengesetzten Ausdrücke ist nach Ansicht mancher sogar unendlich; darüber kann man sich streiten, auf jeden Fall gibt es keine klar angebbare Grenze; ich komme noch darauf zurück.

Die Meinungen beginnen zu schwanken, wenn es darum geht, was die charakteristischen Eigenschaften eines Lexikons und einer Grammatik unabhängig von ihren einzelsprachlichen Ausprägungen sind. Ich will einige Annahmen, die relativ unkontrovers sind, skizzieren.

Eine lexikalische Einheit, oft auch Lexem genannt, ist eine Verbindung von (mindestens) drei Arten von Merkmalen. Dies sind:

- phonologische Merkmale, die den Bedeutungsträger konstituieren, z.B. /u:r/

- semantische Merkmale, z.B. "Gerät zur Zeitmessung"

- kategoriale Merkmale, z.B. "ist ein Nomen”, "wird nach Paradigmenklasse 13 flektiert” usw.

Die kategorialen Merkmale bilden das Bindeglied zwischen elementaren und zusammengesetzen Einheiten, d.h. sie beschränken die Möglichkeiten, aus einfacheren Ausdrücken komplexere zu bilden. In bestimmten Fällen können die phonologischen Eigenschaften fehlen (ein Punkt, der 
Anlaß zu mancherlei Kontroversen gibt), und umgekehrt gibt es elementare Einheiten, denen sich nicht so ohne weiteres eine lexikalische Bedeutung zuweisen läßt (z.B. expletives es). Umgekehrt können mit einer lexikalischen Einheit andere Merkmale verknüpft sein, z.B. graphematische in jenen Sprachen, die über ein Schriftsystem verfügen.

Ein wesentlicher Punkt ist, daß manche Lexeme systematisch eine Art Leerstelle in ihren semantischen Merkmalen vorsehen, die in vorgegebener Weise mit Information aus dem Kontext gefüllt werden muß. Dies sind die deiktischen und anaphorischen Elemente, also Wörter wie ich, hier, jetzt, er, letzterer usw. Teile des Lexikons sind also STRUKTURELL auf den Einbezug kontextueller Information angelegt: es geht nicht nur darum, daß die kontextuelle Information in der Kommunikation berücksichtigt wird, sondern es gibt im Ausdruck selbst bestimmte Stellen, an denen diese Information gleichsam andocken muß.

Die grammatischen Regeln werden traditionell in morphologische und syntaktische unterteilt, je nachdem, ob sie innerhalb eines Wortes oder zwischen Wörtern operieren. Es gibt eine Reihe von Grenzfällen, ebenso wie es eine Reihe von Grenzfällen zwischen Lexemen und zusammengesetzten Einheiten gibt. Dies berührt aber nicht das Prinzip.

All dies kann man noch zu den Trivia zählen. Nicht ganz klar ist, ob dies auch für den folgenden Punkt gilt. Er betrifft eine Unterscheidung, die fast immer in der einen oder anderen Form zwischen zwei Arten von Kompositionsregeln gemacht wird. Es gibt Regeln, die unmittelbar auf der lexikalischen Information, insbesondere den kategorialen Merkmalen operieren, und es gibt Regeln, die dazu dienen, den resultierenden Ausdruck in den Kontext zu integrieren. Typische Beispiele für erste sind etwa die folgenden:

- $\quad$ eine Konstituente, die ein Agens ausdrückt, kommt zuerst; diese Regel basiert auf einem semantischen Merkmal.

der Plural eines Nomens der Klasse 13 wird dadurch gebildet, daß /en/ an das Lexem angehängt wird ("Uhren"); diese Regel beruht auf einem kategorialen Merkmal.

-ein Lexem mit dem kategorialen Merkmal "Artikel" und ein Lexem mit dem Merkmal "Nomen" werden - in dieser Reihenfolge - zu einem Ausdruck mit dem kategorialen Merkmal "Nominalphase" verbunden"

usw. Sie alle beruhen auf Merkmalen, die zur Definition des Lexems (oder auch eines bereits zusammengesetzten Ausdrucks) gehören. Dies können semantische, phonologische oder kategoriale Merkmale sein.

Regeln der Kontextintegration gehen darüber hinaus. Typische Beispiele sind etwa:

- Fokusinformation kommt nach Topikinformation

- Ausdrücke, die Information beibehalten, kommen vor Ausdrücken, die neue Information einführen

Lexeme, die Information beibehalten, werden deakzentuiert, d.h. ihrer suprasegmentalen Eigenschaften beraubt

Lexeme, die Information beibehalten, können ihrer segmentalen phonologischen Eigenschaften beraubt werden ("Ellipse")

usw. Solche Regeln der Bildung komplexer Ausdrücke nutzen durchaus kategoriale, semantisch oder phonologische Merkmale; aber sie erschöpfen sich nicht darin. Schließlich ist es keine lexikalische Eigenschaft des Wortes Uhr, ob es beibehaltene oder neue Information ausdrückt. Andere Regeln der Kontextintegration betreffen den illokutiven Status eines Satzes, wenn er in einem bestimmten Kontext zu bestimmten Zwecken geäußert wird. Typische Beispiele dafür sind 
- $\quad$ Fragen werden durch einen Anstieg der Intonation ohne abschließenden Fall markiert

- $\quad$ eine Assertion wird dadurch markiert, daß das finite Element an zweiter Stelle steht und die Intonation mit einem Fall endet

- $\quad$ ein Imperativ wird dadurch markiert, daß der reine Stamm am Anfang steht

und dergleichen mehr. Auch diese Regeln verwerten lexikalische Merkmale - aber nichts in den phonologischen, kategorialen oder semantischen Merkmalen von schweig- legt fest, ob es zur Bildung einer Behauptung, einer Frage oder einer Aufforderung verwendet wird.

Die Unterscheidung zwischen Regeln, die lediglich die semantischen, phonologischen oder kategorialen Merkmale des Ausdrucks betreffen, und solchen, die dazu dienen, den Ausdruck in bestimmter Weise in den Fluß der Kontextinformation einzubetten, wird in der traditionellen Grammatik selten und in der neueren strukturellen nur gelegentlich explizit gemacht. Implizit findet sie sich aber in beiden, und so kann man sie vielleicht doch zu den Trivia sprachlicher Ausdruckssysteme schlagen.

Das Konstruktionsvermögen muß also in der Lage sein, elementare Verbindungen von Bedeutugnsträgern und Bedeutungen zu bilden, und dies in praktisch unbegrenzter Zahl; es muß in der Lage sein, nach festen Regeln komplexere Ausdrücke zu bilden, und dies gleichfalls in praktisch unbegrenzter Zahl. Schließlich muß es in der Lage sein, einfache und komplexe Ausdrücke so zu bilden, daß sie strukturell auf den Einbezug anderer Information ausgelegt sind. Das Kopiervermögen muß in der Lage sein, der Kommunikation mit anderen zu entnehmen, wie beides in einem bestimmten vorgegebenen System funktioniert, und dies zu replizieren.

Wenn die "Universalgrammatik" nur diese Eigenschaften aufweisen würde, dann wäre dies gut für die Sprachschöpfung, also den ersten der beiden Wege, der vom Sprachvermögen zu einem sprachlichen System führt, denn sie ließe jenen, die ein solches System schaffen, alle Möglichkeiten offen. Hingegen wäre es schlecht für den Spracherwerb, also den zweiten Weg, denn sie würde dem Sprachenlerner kaum eine Handhabe geben, wonach er suchen soll. Gibt es noch andere Eigenschaften, die das Konstruktionsvermögen von Anfang an beschränken und die demnach in bestimmten strukturellen Beschränkungen seines Resultats, eben der einzelsprachlichen Systeme, ihren Niederschlag finden? Das wird generell angenommen, aber die meisten Kandidaten für solche universale Beschränkungen sind umstritten. ${ }^{4}$

${ }^{4}$ In der neueren generativen Grammatik ist die Kombinatorik im wesentlichen auf zwei sehr allgemeine Operation, MERGE und MOVE, begrenzt. Letztere besagt im wesentlichen, daß zwei Ausdrücke (verstanden als Bündel von Merkmalen) miteinander verbunden werden können, letztere, daß die Bündelung von Merkmalen selektiv aufgegeben wird und beispielsweise die phonologischen Merkmale einer Einheit an anderer Stelle auftauchen als die semantischen. Zusätzliche Beschränkungen werden nicht ausdrücklich ausgeschlossen, aber für unökonomisch gehalten und auch nicht vorgeschlagen. Es wird allerdings angenommen, daß MERGE rekursiv ist und damit, daß die Zahl der bildbaren Ausdrücke unendlich ist (Chomsky, Hausser und Fitch 2002). Das ist allerdings keine zusätzliche Beschränkung, es wäre aber eine wesentliche Eigenschaft des Konstruktionsvermögens. In der Tat ist schwer anzugeben, ob es eine maximale Länge für Sätze (diese Ausdrücke sind hier gemeint) gibt. Man beachte, daß Rekursivität im technischen Sinne eine identische Wiederholung gleicher Strukturen erfordert, also nicht nur beispielsweise die beliebige Einbettbarkeit von Nebensätzen, sondern das wiederholte Vorkommen exakt desselben Nebensatzes. Das ist in der Tat möglich, aber es ist schwer zu sagen, ob man dies wirklich als ein konstitutives Merkmal von Sprachssystemen ansehen soll, jedenfalls dann, wenn man der Ansicht ist, daß die resultierenden Strukturen auch von anderen kognitiven Modulen interpretierbar sein sollen. Ein Satz mit $10^{99}$ Wörtern - immer noch sehr endlich - ist von keinem bekannten kognitiven Modul zu interpretieren. Wenn man also ernsthaft der Meinung ist, daß die Rekursivität ein charakteristisches Merkmal des menschlichen 


\subsection{Gleich: einige Nichttrivia}

Weitere Beschränkungen können sowohl die elementaren Einheiten wie die kombinatorischen Regeln betreffen. Es gibt sicherlich nur eine begrenzte Anzahl phonologisch von relevanten Merkmalen, die zum Aufbau von Phonemsystemen genutzt werden können. Die Phonemsysteme der einzelnen Sprachen unterscheiden sich demnach zwar, aber in gewissen, durch die Beschaffenheit der peripheren Organe vorgegebenen Grenzen. Gibt es ähnliche Beschränkungen für die semantische Seite, also für mögliche Wortbedeutungen? Anders gesagt, gibt es "unmögliche Bedeutungen"? Wenn ja, dann würde dies zwar das Potential des menschliche Sprachvermögen einschänken. Aber wir würden es es eher als eine Begrenzung der menschlichen Kognition überhaupt auffassen, nicht so sehr der Möglichkeit, Sprachsysteme zu entwickeln oder zu kopieren. Sicher ist nur, daß eine jede Sprache nur einen geringen Teil der Möglichkeiten nutzt, die uns die Kognition hier zur Verfügung stellt. Das läßt aber offen, ob es grundsätzliche universale Beschränkungen gibt.

Wesentlich schwieriger ist die Argumentation bei den kategorialen Merkmalen. Traditionell gelten gewisse Wortklasseneinteilungen als universal, beispielsweise die Unterscheidung zwischen Verben und Nomina. Allerdings haben sich alle Versuche, eine entsprechende universale Definition zu geben, als wenig befriedigend herausgestellt. Im allgemeinen werden Nomina mit Objekten, Verben hingegen mit Handlungen in Verbindung gebracht. Aber zum einen sind Nomina keine Nominaphrasen; sie referieren daher nicht auf Objekte, sondern sie geben allenfalls Eigenschaften von Objekten an (für Verben gilt entsprechendes). Zum zweiten stimmt dies in vielen Fällen nicht (Fall, Zug, Berechnung). Zum dritten wären dies semantische, keine kategorialen Eigenschaften: ein rein kategeoriale Definition hat noch nie jemand vorgeschlagen. Die Frage "Gibt es eine universale lexikalische Kategorie [+N] bedeutet ja eigentlich: "Gibt es eine Art von lexikalischer Einheit, die sich hinsichtlich ihrer kombinatorischen Möglichkeiten in allen Sprachen gleich verhält?". Eine Antwort darauf setzt voraus, daß die kombinatorischen Möglichkeiten aller Ausdruckssysteme entweder gleich oder zumindest hinlänglich ähnlich sind. Damit ist das Problem von möglichen lexikalischen Universalien zu möglichen kombinatorischen Universalien verschoben.

In früheren Versionen der generativen Grammatik gibt es eine Fülle von Vorschlägen in diese Richtung. Dazu zählen beispielsweise Beschränkungen der Phrasenstruktur, die allesamt dem "X-bar-Schema" folgen; weiterhin zählen dazu universale - gegebenenfalls parametrisierte Beschränkungen auf mögliche Transformationen, wie sie seit der Dissertation von John Robert Ross im Jahre 1967 in verschiedenen Formen ausgearbeitet wurden, oder (strukturelle!) Beschränkungen für mögliche Koreferenz zwischen zwei Ausdrücken, wie sie in der Chomskyschen Bindungstheorie vorgeschlagen wurden (Chomsky 1981). Keine dieser Beschränkungen ist jedoch allgemein akzeptiert, und so sind sie denn auch im Minimalismus fürs erste einmal aufgegeben.

Es gibt eine Reihe ganz anderer Vorschläge. Wunderlich (2003, S. 5ff) beispielsweise gibt die folgende Kandidatenliste von "basic characteristics of human language":

"(i) Symmetry: Language is a speaker-hearer symmetric system. This concerns pragmatics as well as linguistic processing. [...]

Sprachvermögens ist, dann muß man zugleich annehmen, daß fast alle Strukturen aller menschlichen Sprachen nicht interpretierbar sind ("fast alle" im üblichen mathematischen Sinn von "alle außer endlich vielen"). 
(ii) Off-line processing: Utterances can refer to situations that are not present. This concerns semantics. [...]

(iii) Discreteness (or digitization): Minimal change of features yields a different meaning. This primarily concerns segmental phonology. [...]

(iv) Double articulation: Minimal elements are meaningless, only larger elements bear meaning. This concerns the important difference between lexicon and phonology. Double articulation allows to create a larger inventory of lexical units by minimal means.

(v) Duality: Partws of utterances are memorized, thus constituting the lexicon, while complete utterances are produced combinatorily, according to morphosyntactic rules or principles. [...]

(vi) Reference and predication: Any meaningful utterance about a state of affairs expresses some predication that involves potential referents, so in addition it must be referentially anchored. Combinatorial systems have two clear advantages in terms of economy and flexibility. They reduce the expense of storage (economy), and allow small combinatorial changes to bring about large effects (flexibility)."

All dies scheint mir im Prinzip richtig. Die beiden ersten Charakteristika und teilweise auch das letzte beziehen sich allerdings eher auf die Funktion von Sprachsystemen in der Kommunikation als auf ihre Struktur; die Charakteristika (iii) - (v) entsprechen dem, was oben in Abschnitt 4.2 als Gemeingut aller Sprachwissenschaftler bereits genannt wurde. Ein wesentlicher Zusatz sind lediglich "Referenz und Prädikation", genauer gesagt, der Umstand, daß alle Sprachen Teilsysteme ausbilden, die diese beiden Funktionen für kommunikative Zwecke zulassen. In der Tat verfügen alle menschlichen Sprachen über diese beiden Möglichkeiten. Sie lassen sich allerding nicht durch rein formale universale Beschränkungen auf die Kombinatorik definieren, in jenen Sinne, in dem beispielsweise das X-bar-Schema eine universelle Beschränkung auf die Form von Ausdrücken ist. Alle Sprachen erlauben verschiedene Formen der Referenz. Aber die Teilsysteme von Ausdrücken, die sie dazu entwickeln, sind strukturell nicht gleich - wenn auch oft ähnlich, vor allem natürlich, wenn die betreffenden Sprache genetisch verwandt sind.

Referenz und Prädikation exemplifizieren in meinen Augen die einzig plausible Form von universalen Eigenschaften menschlicher Sprachen, die nennenswert über die Trivia hinausgeht. Dies sind strukturell verankerte Funktionen. Was damit gemeint ist, will ich im folgenden Abschnitt kurz erläutern und dann an einem Beispiel, der Finitheit, eingehender betrachten.

\subsection{Strukturell verankerte Funktionen als Universalia}

Ausdruckssysteme - das Produkt unseres Sprachvermögens - müssen zur Kommunikation tauglich sein. Sonst kämen sie nicht zustande - weder auf dem Wege der Sprachschöpfung noch dem des Spracherwerbs. Dies leisten sie, indem sie bestimmte Funktionen erfüllen, und dazu wiederum müssen sie bestimmte strukturelle Teilsysteme ausbilden, die das Konstruktionsvermögen möglich macht. Diese Teilsysteme müssen allerdings nicht unbedingt in allen Sprachen realisiert und schon gar nicht in gleicher Weise. Sie können also sehr unterschiedlich ausfallen, vor allem aber ganz fehlen. Man muß also, um eine alte Unterscheidung aufzugreifen, universalia potentia (dynamei) und universalia actu (energeia) unterscheiden - solche, die der Möglichkeit nach bestehen, weil das menschliche Konstruktionsvermögen sie bilden kann, und solche, die tatsächlich bestehen, weil es sie in der Kommunikation ausgearbeitet hat.

Wenn das Konstruktionsvermögen tatsächlich eine solche strukturell verankerte Funktion entwickelt, dann bedient es sich dazu bestimmter Mechanismen, die im strukturelle Potential eines Ausdruckssystems vorgegeben sind. Zu diesem Potential zählen zunächst einmal die obengenannten Trivia. Diese wiederum eröffnen weitere Möglichkeiten, beispielsweise das, was man traditionell als SKOPUS bezeichnet. Wenn ein Ausdruck zusammengesetzt ist und ein weiterer Ausdruck hinzugefügt wird, dann kann dies - je nachdem, wie der Ausgangsausdruck 
beschaffen ist - an verschiedenen Stellen geschehen. Je nachdem ist der Effekt ein unterschiedlicher. Ich will dies an einem elementaren Beispiel, der Negation durch die Partikel nicht, erläutern. Ganz allgemein wird angenommen, daß diese Partikel den Wahrheitswert des Satzes, in den sie eingefügt wird, umdreht. Sie kann aber an verschiedenen Stellen eingefügt werden; das hat Konsequenzen:

(1) a. Gestern ist Maria zum ersten Mal nicht gekommen.

b. Gestern ist Maria nicht zum ersten Mal gekommen.

In beiden Fällen bringt der Sprecher zum Ausdruck, daß der Satz Gestern ist Maria zum ersten Mal gekommen falsch ist. Das ist aber nicht alles. Mit der Äußerung von 1a bringt er darüber hinaus, gleichsam in einer sekundären Assertion, zum Ausdruck

daß Maria gestern nicht gekommen ist, und

$\mathrm{da}$ es eine Reihe von Gelegenheiten zuvor gegeben hat, zu denen sie gekommen ist, und daß gestern die erste ist, für die dies nicht zutrifft.

Dies ist anders in 1b. Hier ist offen, ob sie gestern gekommen ist oder nicht. Aber wenn sie gestern gekommen ist, dann muß sie schon zu früheren Gelegenheiten gekommen sein.

Dies sind nur zwei von verschiedenen Möglichkeiten, die Negation zu plazieren; man kann auch sagen Nicht gestern ist Maria zum ersten Mal gekommen oder Gestern ist nicht maria zum ersten Mal gekommen, und je nachdem variiert der Effekt systematisch. Das Deutsche ist hier besonders flexibel; aber auch andere Sprachen nutzen die Möglichkeit, den Skopus bestimmter Elemente zu ändern. Diese Möglichkeit beruht auf einer elementaren Eigenschaft von Ausdruckssystemen, die sich aus ihrer Kombinatorik ergibt: die einzelnen Ausdrücke sind sequentiell geordnet. Dies zählt zu den Trivia, die oben in Abschnitt 4.1 genannt wurden. Diese Trivia machen die Entfaltung weiterer struktureller Mittel, die jeweils bestimmte funktionale Zwecke erfüllen, möglich. Wir wissen nicht, wann in der Geschichte der menschlichen Sprachentwicklung jemals jemand zum ersten Mal von dieser Möglichkeit gebraucht gemacht hat. Es ist ein "universale potentia", das inzwischen in allen uns bekannten Sprachen zu einem "universale actu" umgesetzt wurde und demnach auch von allen Sprachlernern kopiert werden kann. Diese Umsetzung ist eine Entwicklung, der sich in der Kommunikation vollzogen hat und, im Falle des Spracherwerbs, nach wie vor ständig vollzieht.

Es genügt, wenn das Konstruktionsvermögen selbst nur einfache Systeme zustande bringt, jene, die den Trivia genügen - ein Vorgang, der selbst von Anfang an das Kommunikationsvermögen erfordert. Die funktionalen Anforderungen der Kommunikation führen dann zu einem weiteren Ausbau der strukturellen Möglichkeiten von Ausdruckssystemen. Manche davon werden in allen Sprachen realisiert, manche in vielen, manche in wenigen. Die weiter oben schon erwähnte Subordination ist ein anderes Beispiel; es ist wiederholt bemerkt worden (Schumann u.a., im Druck), daß Sprachen, für die es keine Schriftkultur gibt, oft nur wenige subordinatierte Strukturen aufweisen - wenn überhaupt. Die deutet darauf, daß es erst mit der Schriftsprache zur Ausbildung dieser Konstruktion - und damit der wichtigsten Quelle der Rekursivität - gekommen ist. Die Subordination liegt also sehr wohl im konstruktiven Potential des menschlichen Sprachvermögens; aber sie hat sich erst auf der Basis anderer Strukturen geformt, wesentlich gesteuert durch Formen der Kommunikation, die ein anderes Medium als der flüchtige Schall eröffnet hat.

Wenn dies so ist, dann es mag durchaus sein, daß strukturell verankerte Funktionen von Ausdruckssystemen denkbar sind, die bislang in keiner uns bekannten Sprache verwirklicht wurden, die aber durch das Zusammenwirken von Konstruktionsvermögen und Kommunikationsvermögen entwickelt werden könnten. So wird oft argumentiert, daß es in den natürlichen Sprachen keine "numerischen Regeln" gibt, also Regeln der Art "Stelle die vierte 
Konstituente ans Ende des Satzes". Dies ist, von marginalen Ausnahmen abgesehen, in der Tat so. Aber man mag sich fragen, ob diese Möglichkeit vom Sprachvermögen - von der Universalgrammatik - vorgegeben ist oder ob sie nur noch nicht verwirklicht wurde, weil entsprechende strukturelle Teilsysteme nicht erforderlich sind.

Im nächsten Abschnitt werde ich eine strukturell verankerte Funktion natürlicher Sprachen etwas eingehender betrachten, die zwar auf eine lange Geschichte in der grammatischen Kunst zurückblicken kann, die aber dort immer ein etwas stiefmütterliches Dasein geführt hat. Dies ist die FINITHEIT, im Sinne des Unterschieds zwischen finiten und infiniten Verbformen.

\section{Finitheit}

\subsection{Was heißt "finit”?}

Die Finitheit ist im vorliegenden Zusammenhang aus zwei Gründen besonders interessant. Zum einen findet sie sich nach allgemeiner Ansicht in den meisten Sprachen der Welt, aber nicht allen. Chinesisch (sowohl Mandarin wie Kantonesisch) ist die bekannteste, aber nicht die einzige Ausnahme (siehe z.B. Duffield (im Druck) zum Vietnamesischen und zur Finitheit in solchen Sprachen allgemein). Die Finitheit ist ein Fast-Universale. Zweitens ist auffällig, daß Lerner sie zunächst zu vernachlässigen scheinen. Die ersten Äußerungen wie Erstspracherwerb von Kindern wie auch im (ungesteuerten) ${ }^{5}$ Zweitspracherwerb von Erwachsenen zeigen vorrangig oder gar ausschließlich nicht-finite Strukturen. Dies gilt auch, wenn die zu lernende Sprache (und, beim Zweitspracherwerb, die Ausgangssprache) reich an finiten Formen sind. Im ungesteuerten Zweitspracherwerb durchlaufen die Lerner regelhaft mehrere Entwicklungsphasen (Klein und Perdue 1989, 1997). Die erste ist eine "nominale Struktur" gekennzeichnet, d.h. die Äußerungen bestehen in erster Linie aus Nomina (oder festen Wendungen); zwar treten Verben auf, aber sie verhalten sich eher wie Nomina, d.h. sie zeigen keine oder kaum Rektionseigenschaften, und sie sind nicht flektiert. Es gibt kaum strukturelle Organisationsprinzipien. In der zweiten Phase, der "Basic Variety", werden zwar systematisch Verben verwendet, sie zeigen Rektionseigenschaften, sind aber nicht flektiert ${ }^{6}$ : es handelt sich um eine verbale, aber nichtfinite Form des Äußerungsaufbaus. Im übrigen gibt es einige klare Strukturprinzipien, die die Form möglicher Sätze beschränken. Erst in der dritten Phase, die nur von einem Teil der Lerner erreicht wird, zeigt sich die für Ausgangs- und Zielsprache charakteristische Form des finiten Äußerungsaufbaus.

Daß Kinder zu Beginn vorwiegend nichtfinite Formen verwenden, ist seit langem bekannt. Diese Beobachtung hat aber erst seit Beginn der Neunzigerjahre unter dem Stichwort "root infinitives" eine erhebliche Aufmerksamkeit auf sich gezogen; einen guten Überblick über die lebhafte Diskussion vermitteln Dimroth und Lasser 2002, Blom 2001. Die resultierenden Strukturen sind nicht unbedingt dieselben wie beim Zweitspracherwerb; es besteht aber kein Zweifel, daß es sich um verwandte Phänomene handelt: die Finitheit wird anfangs nicht oder jedenfalls nicht regelhaft markiert.

${ }^{5}$ Für den gesteuerten, d.h. durch Unterricht vermittelten Spracherwerb gilt dies nicht. Das liegt daran, daß den Lernern die Flexionsmorphologie und damit die finiten Verbformen von Anfang an eingebleut werden.

${ }^{6}$ Dies schließt nicht das Vorkommen von Formen aus, die in der jeweiligen Zielsprache als finit gelten würden. Aber diese Formen werden nicht funktional variiert, d.h. eine Form wie geht wird verwendet wie gehen oder gegangen. 
Was aber heißt Finitheit? Der Begriff stammt von Priscian, dem grammatischen Lehrmeister des Abendlandes; er wendet ihn jedoch gleichermaßen auf Verben wie auf Nomina an. Finite Formen sind demnach solche, die sich auf einen konkreten Fall beziehen, also auf ein bestimmtes Objekt oder eine Menge von Objekten, oder auf eine bestimmte Handlung oder Menge von Handlungen. Infinite Formen leisten dies nicht, sondern sie geben lediglich Eigenschaften von irgendwelchen Entitäten an. In der Folge hat sich der Begriff auf finite (und entsprechend) nicht-finite Verbformen verengt. Er wird aber so gut wie nie definiert, sondern lediglich exemplarisch anhand bestimmter Flexionsformen eingeführt: amor, amabis, amavisset, amavisti sind finite Formen, amare, amata, amans, amavisse sind infinite Formen. Es liegt daher nahe, die Finitheit als Kategorie der Verbalflexion und damit der Flexionsmorphologie zu sehen. Dies ist die gängige Vorstellung, und auch in den zahllosen Arbeiten zum Erwerb der Finitheit ist es nicht anders. Sie stößt aber auf zwei substantielle Probleme.

Erstens wird die Unterscheidung zwischen finiten und infiniten Formen auch bei Sprachen getroffen, in denen so gut wie keine morphologische Markierung am Verb erfolgt. Englisch ist ein gutes Beispiel. Mit ganz wenigen Ausnahmen wie z.B. has oder swam können alle "finiten" Formen auch infinite Formen sein; umgekehrt gibt es außer dem Partizip Präsens und einigen seltenen irregulären Formen wie swum kaum eine infinite Form, die auch nicht finit verwendet werden könnte. Dennoch betrachtet jedermann die Form left in He left als finit, und in He has left als nichtfinit. Der Grund kann nicht an in der Flexionsmorphologie liegen. Einige Modalverben wie should oder can werden unweigerlich als finit angesehen, obwohl sie morphologsich überhaupt nicht markiert sind. Auch hier muß es also einen anderen Grund als die Flexion geben. Was ist dieser Grund? Damit ist nicht gesagt, dass es nicht eine morphologische Seite der Finitheit gibt; in vielen Sprachen wird die Finitheit durch Flexion ausgedrückt. Aber zwingend daran gebunden ist sie nicht: die Finitheit ist keine rein morphologische Kategorie.

Zweitens gibt es eine Fülle von syntaktischen, semantischen und pragmatischen Gegebenheiten, die mit der Finitheit einhergehen. Die offenkundigste ist die "Finitheitsbeschränkung" in allen Sprachen, die die Finitheit überhaupt markieren:

(2) Eine komplexe Verbform kann mehrere infinite, aber nur eine finite Form enthalten.

Man kann im Deutschen sagen Er muß singen können, nicht aber im Englischen He must can sing. Dies hat keine semantische Gründe; man kann durchaus sagen He must be able to sing. Aber can ist eine finite Form, und da must auch eine finite Form ist, ist ein solcher Satz ausgeschlossen. Was ist der funktionale oder strukturelle Grund für diese Beschränkung?

Die Finitheitsbeschränkung ist nur einer der Effekte, die mit der Finitheit verknüpft sind. Im folgenden gehe ich durch eine Reihe von syntaktischen, semantischen und pragmatischen Gegebenheiten, die allesamt irgendwie von der Finitheit abhängen.

\subsection{Syntaktische Eigenschaften der Finitheit}

Im Deutschen gibt es eine alte Diskussion um die Grundwortstellung. Die zentralen Fakten sind klar und lassen sich durch drei Regeln beschreiben:

(3) a. In deklarativen Hauptsätzen steht das Verb nach der ersten Hauptkonstituente $\left(V_{2-}\right.$ Regel).

b. In Nebensätzen steht das finite Verb zuletzt $\left(V_{\text {letzt }}\right.$

c. In Entscheidungsfragen und in Imperativen steht das Verb am Anfang $\left(V_{1}\right)$.

Die Diskussion geht im wesentlichen um die Frage, ob die Grundwortstellung (in Hauptsätzen) SOV oder SVO ist. In dieser Form ist die Frage aber nicht sinnvoll, denn es kommt darauf an, 
ob man $\mathrm{V}$ als finite oder als infinite Verbform betrachtet. Ein deutscher Satz kann viele Verbformen enthalten, aber nur eine finite. In Formen wie kam oder schlief sind finite und lexikalische Komponente des Verbs in zu einer Form verschmolzen. Welche dieser beiden Komponenten ist für die Wortstellung verantwortlich? Es ist offenbar die Finitheit, wie sofort deutlich wird, wenn man beide Komponenten auf zwei Formen verteilt:

(4) a. Gestern ist Maria zum ersten Mal gekommen.

b. ..., obwohl Maria gestern zum ersten Mal gekommen ist.

c. Ist Maria gestern zum ersten Mal gekommen!

Deutsch ist also weder SOV noch SVO, or VSO - es ist "FIN-Zweit". FIN benutze ich hier und im folgenden als Abkürzung für "Finitheit" und, wo kein Mißverständnis zu befürchten ist, "jenes Element, das die Markierung der Finitheit trägt". Entsprechendes gilt für die anderen Satztypen: Nebensätze sind normaleweise "FIN-letzt", Entscheidungsfragen und Imperative "FIN-erst". Dies deutet auf einen anderen wichtigen Punkt: offenkundig gibt es einen engen Zusammenhang zwischen der Finitheit, genauer gesagt, ihrer Position, und der illokutiven Rolle eines Satzes. Wir können diese Beobachtungen folgendermaßen zusammenfassen:

(5) a. Die Grundwortstellung des deutschen Satzes hängt von der Stellung der Finitheit ab und nicht von der des lexikalischen Verbes.

b. Ein Satz kann keine Assertion ausdrücken, wenn FIN nicht an zweiter Stelle steht.

Dies ist nicht das einzige wichtige syntaktische Faktum, das gewöhnlich mit dem Verb in Zusammenhang gebracht wird, aber eigentlich von der Finitheit abhängt und nur indirekt vom Verb als lexikalischer Einheit. Ein anderes ist eine Ellipsenregel, die seit von John Robert Ross "Gapping" getauft wird, weil die gleichsam ein Loch mitten im Satz schafft. Sie besagt, daß ein identisches Verb in einer Koordination beim zweiten Vorkommen weggelassen werden kann. Auch hier ist leicht zu sehen, daß es nicht auf das Verb, sondern auf FIN ankommt (identische Elemente sind kursiv):

(6) a. Nikos schlief im Bett und Melitta (schlief) auf dem Sofa.

b. Nikos hat im dem Bett geschlafen und Melitta (hat) auf dem Sofa gedöst

c. Nikos hatte im dem Bett geschlafen und Melitta hat auf dem Sofa*(geschlafen).

d. Nikos hat im Bett geschlafen und Melitta (bat) auf dem Sofa (geschlafen).

Die Regel des Gapping ist also kein Fall von Verb-Ellipse, sondern von "FIN-Ellipse". Ein lexikalisches Verb allein kann nicht im zweiten Konjunkt weggelassen werden, wenn es mit einem Verb in entsprechender Position im erste Konjunkt identisch ist. Falls FIN und lexikalisches Verb in einer Form zusammenfallen, wie in 6a, ist Gapping natürlich möglich.

Ein anderer syntaktischer Effekt, der eng mit FIN zusammenhängt, betrifft die "Lizenz", d.h. den Umstand, daß ein bestimmtes Elemente nur vorhanden sein kann, wenn ein anderes vorhanden ist, von dem es "lizensiert" wird. Hier gibt es im Deutschen zwei klare Regeln?:

(7) a. Kein grammatisches Subjekt ohne Finitheit.

7Die Begriffe "Subjekt" und "expletives Element" werden in der Linguistik nicht ganz einheitlich verwendet; deshalb sind die beiden folgenden Behauptungen mit etwas Vorsicht zu lesen. Insbesondere ist im Deutschen nicht jedes es ein Expletiv. Hier gemeint ist das informationsstrukturelle es, wie in Es hat jemand angerufen, nicht das vom Verb regierte es, wie in Es hat gehagelt. 
b. Kein expletives Element ohne Finitheit.

Es gibt zwar auch infinite Sätze, wie z.B. das Komplement in (Ulli beschloß), nie wieder Maria Blumen zu schenken. Aber das (implizite) Subjekt Ulli kann nicht als grammatisches Subjekt realisiert werden. Von dieser Beschränkung gibt es einige interessante Ausnahmen, wie etwa:

(8) a. Ich eine Krawatte tragen - niemals!

b. Ich und eine Krawatte tragen - niemals!

Diese Sätze haben einen eigentümlichen illokutiven Status - sie machen keine Behauptung; aber sie stellen auch keine Frage; eine Frage fordert den Angesprochenen zu einer Antwort auf, und das ist hier nicht der Fall. Sie geben eher den Eindruck, als würde irgendein Sachverhalt nur genannt, in 8a,b der Sachverhalt, daß der Sprecher eine Krawatte anzieht: Sie führen eine "Topik" ein und lassen offen, was darüber gesagt wird. Insbesondere wird keine illokutive Rolle zum Asudruck gebracht. Dies bestätigt den schon erwähnten Zusammenhang zwischen FIN und der Illokution eines Satzes (vgl. 4 und 5).

Diese wenigen Beobachtungen zur Syntax machen zweierlei deutlich. Erstens ist die Finitheit nicht einfach eine Frage der Verbflexion, sondern sie greift tief in die Struktur des Satzes, hier des deutschen Satzes, ein. Zweitens hat die Finitheit offenbar etwas mit der Informationsstruktur und der illokutiven Rolle des Satzes, insbesondere mit einem möglichen assertiven Charakter, zu tun.

Wenn dies zutrifft, dann könnte es so etwas wie "Finitheit" sehr wohl auch in Sprachen geben, die nach üblicher Vorstellung keine Finitheit kennen, wie Chinesisch oder Vietnamesisch. Man muß hier klar zwischen der Kategorie der Finitheit selbst und einer bestimmten Art, wie sie markiert wird, trennen. Letzteres erfolgt in vielen Sprachen über die Verbflexion, und das ist sicher kein Zufall. Aber es ist nicht die einzige Möglichkeit.

\subsection{Semantische Eigenschaften der Finitheit}

\subsubsection{Spezifische und nichtspezifische Interpretation von Nominalphrasen}

Traditionell unterscheidet man definite Nominalphrasen wie der Freund, dieser Freund von indefiniten wie ein Freund, drei Freunde. Letztere können eine spezifische und eine nichtspezifische Lesart haben. Manche Verben erzwingen eine spezifische Lesart, wenn die Nominalphrase in Objektposition steht, andere lassen dies offen:

(9) a. Melibos traf einen Freund.

b. Melibos suchte einen Freund.

In 9b kann kann Melibos nach einem bestimmten Freund gesucht haben, z.B. Leander, oder aber er war auf der Suche nach irgendjemanden, wenn es nur ein Freund ist. In 9a ist hingegen nur die erste dieser beiden Lesarten möglich. Da die beiden Sätze sich nur durch die semantischen Merkmale der Verblexeme treff- und such- unterscheiden, wird der Unterschied gewöhnlich mit dem Unterschied zwischen zwei Verbtypen, damit zwei Arten von semantischen Merkmalen in Zusammenhang gebracht: Verben wie treff- sind "transparent", Verben wie suchsind opak; erstere werden als Normalfall angesehen. Dieses Phänomen hat in der formalen Semantik große Aufmerksamkeit gefunden (siehe z.B. Zimmermann 1993).

Die beiden Verben in 9 sind finit, d.h. sie verschmelzen das lexikalische Verb mit FIN zu einr Form. Merkwürdigerweise verschwindet der Unterschied, wenn man FIN wegläßt:

(10) a. Es ist schön, einen Freund zu treffen. 
b. Einen Freund zu treffen, passiert einem ja nicht selten.

Hier hat die Nominalphrase eine spezifische wie eine nichtspezifische Lesart, obwohl das Verb "transparent" ist. Der Unterschied taucht aber oft wieder auf, wenn die indefinite Nominalphrase im Skopus von FIN steht; es muß aber nicht der Fall sein:

(11) a. Melibos gelang es, einen Freund zu treffen.

b. Melibos wünschte, einen Freund zu treffen.

Offenbar gibt es also einen klaren Zusammenhang zwischen spezifischer Lesart und Finitheit, den man so formulieren kann:

(12) Indefinite Nominalphrasen können nur dann eine spezifische Lesart haben, wenn sie direkt oder indirekt im Skopus von FIN stehen.

Man betrachte nun die beiden folgenden Sätze:

(13) a. Dreimal kaufte Melibos einen Wagen.

b. Melibos kaufte einen Wagen dreimal.

Im ersten Fall wird über drei verschiedene Zeiten geredet - sagen wir die Zeiten T1, T2 und T3. Sie liegen allesamt in der Vergangenheit, und über jede von ihnen wird gesagt, daß Melibos einen Wagen gekauft hat. Es gibt also einen T1-Wagen, einen T2-Wagen und einen T3-Wagen, den Melibos gekauft hat. Dies könnte bei allen drei Gelegenheiten derselbe sein, er muß es aber nicht, und es ist, jedenfalls in diesem Fall, auch unwahrscheinlich. Die Spezifik ist also relativ zu den Zeiten, über die eine Behauptung gemacht wird - sie ist relativ zu den "Topikzeiten", wie ich sagen will. "Spezifisch" heißt "singulär in Bezug auf eine Topikzeit".

Im zweiten Fall, der sich nur durch die Position der Angabe dreimal unterscheidet, wird hingegen nur eine Behauptung über eine möglicherweise lange Zeit in der Vergangenheit gemacht, von der gesagt wird, daß sie drei Kaufsituationen enthält; hier muß der gekaufte Wagen derselbe sein, weil nur über eine Topikzeit eine Behauptung gemacht wird. Deshalb sind Sätze wie $13 \mathrm{c}$ auch merkwürdig, weil man gemeinhin nicht dasselbe Bier dreimal trinken kann:

(13) c. Melibos hat ein Bier dreimal getrunken.

Was hat dies nun mit der Finitheit zu tun? Nichtfinite verbale Ausdrücke wie einen Freund treffen oder einen Wagen kaufen sind selektive Beschreibungen von Situationen (sie geben z.B. kein Agens an). Aber sie beziehen die beschriebene Situation(en) des Freund-Treffens oder WagenKaufens nicht auf eine Topikzeit, also eine Zeit, über die eine Assertion getroffen wird. Es wird daher auch auch nicht behauptet, daß die beschriebene Situation zu irgendeiner Gelegenheit in der Vergangenheit, Gegenwart oder Zukunft der Fall ist. Daher ist die indefinite Nominalphrase auch nicht "Topikzeit-singulär", also nicht spezifisch. Wenn sie dennoch als spezifisch verstanden wird - eine Möglichkeit, die durchaus besteht -, dann hat dies keine strukturellen Gründe, sondern es liegt am Weltwissen des Sprechers oder Hörers oder an irgendwelchen Kontextinformationen.

\subsubsection{Tempus}

In der sprachwissenschaftlichen Tradition wird das Tempus regelmäßig mit der Finitheit eines Verbs in Verbindung gebracht. Nichtfinite Formen können zwar durchaus Zeit ausdrücken (man 
denke an den Unterschied zwischen schlafen und geschlafen haben), jedoch kein Tempus. Das Tempus im klassischen Verstand ist eine deiktische, zeitrelationale Kategorie des Verbs, die dike beschriebene Situation relativ zur Äußerungszeit einordnet. In Der Papst war krank wird das Kranksein des Papstes vor die Sprechzeit gelegt, in Der Papst wird krank hinter die Sprechzeit, und in Der Papst ist krank wird angegeben, daß die Sprechzeit in die Zeit des Krankseins fällt. Dies is so fest etabliert, daß man sich fast geniert, es so ausdrücklich anzuführen. Es ist aber nur manchmal richtig. Beispiele wie das folgende machen dies deutlich:

$$
\text { (Warum hat der Papst den gestern keine Audienz gegeben?) - Er war krank. }
$$

Hier wird keineswegs gesagt, daß sein Kranksein vor der Sprechzeit liegt. Es kann durchaus sein, daß er zur Sprechzeit immer noch krank ist. In Der Papst war tot ist es fast sicher so, daß er zur Sprechzeit noch tot ist. Das Präteritum war drückt vielmehr aus, daß eine bestimmte Zeit, über die aus irgendeinem Grunde etwas gesagt wird - in 14 z.B. die Zeit, zu der er eine Audienz hätte geben sollen - vor der Sprechzeit liegt. Über diese Topikzeit ist dann etwas gesagt, nämlich daß zu dieser Zeit der Papst krank oder auch tot war. Die Situationszeit - also die Zeit, zu der der Papst krank oder tot ist - kann aber viel länger dauern; deshalb kann oder muß sie sogar die Sprechzeit einschließen, also in jener Relation zur Sprechzeit stehen, die man gemeinhin dem Präsens zuweist.

Die Funktion des Tempus ist es vielmehr zu markieren, ob die TOPIKZEIT der Sprechzeit vorausgeht, folgt, oder sie enthält. Die Situationszeit wiederum kann der Topikzeit folgen, vorausgehen, sie enthalten oder auch, in anderen Fällen, in ihr enthalten sein. Man hat es also mit zwei zeitlichen Relationen zu tun: Sprechzeit zu Topikzeit und Topikzeit zu Situationszeit; die Relation zwischen Sprechzeit und Situationszeit ist nie direkt, sondern immer zusammengesetzt. Die erstere entspricht dem Tempus, die letztere dem, was man traditionell den (grammatischen) Aspekt nennt. Beide werden in vielen Sprachen durch die Verbmorphologie markiert; das muß aber nicht der Fall sein. Man kann sich dies an einem einfachen Beispiel vor Augen führen, dem aspektuellen Unterschied zwischen den beiden englischen Präteritalformen John closed the window und John was closing the window. In beiden Fällen plaziert die Tmpusmarkierung die Topikzeit, die Zeit also, über die eine Behauptung gemacht wird, vor die Sprechzeit. Man kann den intuitiven Unterschied zwischen beiden Formen leicht erfassen, wenn man annimmt, daß im ersten Fall die gesamte Situationszeit (grob gesagt: Fenster offen und John dreht am Griff - Fenster zu) in der Topikzeit enthalten ist, während im zweiten Fall die Topikzeit in das erste Teilintervall der Situationszeit fällt, jenes Intervall, in dem das Fenster noch nicht zu ist und John am Griff dreht (oder was immer er tut, um diesen Zustand zu ändern). Im ersten Fall erstreckt sich die Behauptung über die ganze Situation, d.h. das Fenster muß in dieser Zeit geschlossen worden sein: der Satz ist perfektiv. Im zweiten Fall gilt die Behauptung nur einem Teilintervall; es ist nicht behauptet, daß das Fenster dann auch geschlossen wurde - allerdings auch nicht ausgeschlossen. Es liegt einfach jenseits der Zeit, über die eine Behauptung gemacht wird. Deshalb ist John was closing the window imperfektiv. Diese nur grob umrissenen Überlegungen soll hier nicht näher ausgeführt werden (vgl. dazu Klein 1994). Im vorliegenden Zusammenhang geht es lediglich um die natürliche Veerbindung zwischen Tempus und Finitheit.

Das Tempus legt die Topikzeit fest - wenn auch nur grob: es situiert sie relativ zur Sprechzeit. Es ist daher nicht verwunderlich, dass viele Sprachen Tempus und Finitheit in einer Form verschmelzen. Die verschiedenen Finitheitseffekte, die wir im Voranstehenden diskutieren haben, haben nichts direkt mit der Zeit zu tun, so wie sie durch die Tempusmarkierung ausgedrückt wird. Der Zusammenhang ergibt sich vielmehr daraus, dass das Tempus die Assertion auf eine bestimmte Zeit, eben die Topikzeit, beschränkt.

\subsection{Prosodische Eigenschaften der Finitheit}


Wenn man den Bedeutungsbeitrag bestimmen will, den ein Ausdruck zur Gesamtbedeutung der Konstruktion macht, dann ist es oft hilfreich, diesen Ausdruck durch kontrastive Intonation zu markieren. Was passiert, wenn der so hervorgehobene Ausdruck ein finites Verb ist, wie im folgenden Beispiel?

(15) Leander LAG auf dem Sofa.

Intuitiv, der Kontrast kann in drei Richtungen gehen:

(16) a. (Er SASS nicht auf dem Sofa.) Er LAG auf dem Sofa.

b. (Er LIEGT nicht auf dem Sofa.) Er LAG auf dem Sofa.

c. (Leander lag nicht auf dem Sofa.) - Doch, er LAG auf dem Sofa.

In 16a werden die semantischen Merkmale des Lexems lieg- kontrastiert, in 16b das Tempus, das sich aus der Flexion ergibt. Der interessante Fall ist 16c. Hier wird die Assertion selbst kontrastiert: der Gegensatz besteht zwischen "es war der Fall - es war nicht der Fall”. Eine finite Verbform wie lag verschmilzt eine lexikalische Komponente lieg- mit einer finiten Komponente, hier ausgedrückt durch den Ablaut. Die Finitheit ist also zum einen Träger des Tempus, zum anderen Träger der ASSERTION.

Nicht alle finiten Sätze drücken eine Assertion aus; Fragesätze und Nebensätze sind auch finit. In diesem Fall kommen andere illokutive Rollen ins Spiel, etwa Fragen oder Befehle, bei denen, wie wir gesehen haben, der Träger der Finitheit seine Position wechselt. ${ }^{8}$ Oder aber die Assertion wird in bestimmter Weise durch andere Ausdrucksmittel überlagert, in Relativsätzen oder temporalen Nebensätzen. Wie dies im einzelnen zu analysieren ist, will ich hier nicht weiter verfolgen. Stattdessen soll der zentrale Begriff der Topikzeit in einen etwas weiteren Zusammenhang gestellt werden. Bis hierhin haben wir angenommen, dass die Finitheit dazu dient, den nichtfiniten Teil des Satzes, seinen deskriptiven Gehalt gleichsam, zu der Zeit in Bezug zu setzen, über die eine Assertion getroffen wird - zur Topikzeit. Man mag sich nun fragen, ob es hier nur um eine zeitliche Einschränkung der Assertion geht oder ob auch nichtzeitliche Momente ins Spiel kommen.

\subsection{Die Topiksituation}

Ist die folgende Behauptung, hier und jetzt geäußert, wahr oder falsch:

(17) Es gab keinen Wein mehr.

Auch wenn man nicht nur Deutsch kann und somit den Satz versteht, sondern auch weiß, wie die Welt beschaffen war, ist und sein wird - vielleicht sogar alle mögliche Welten - kann man darauf keine sinnvolle Antwort geben. Dazu müßte man wissen, ÜBER WELCHE SITUATION DER SPRECHER REDET. Je nachdem ist die Antwort "ja" oder "nein". Jede Assertion ist relativ zu einer Situation, über die man redet - einer TOPIKSITUATION, wie ich sagen will. Die Topikzeit ist einer, aber nur einer der Parameter, die diese Situation festlegen.

Es gibt verschiedene Wege deutlich zu machen, was die Topiksituation bei einer gegebenen Äußerung ist:

${ }^{8}$ Dies schließt selbstverständlich nicht aus, daß andere Mittel benutzt werden, um illokutive Rollen zu markieren, beispielsweise Partikel oder W-Elemente. 
- $\quad$ in vielen Fällen liefert eine explizite Frage die nötigen Angaben, etwa "Was ist dir gestern abend passiert?" oder "Weshalb waren die Gäste bei der Hochzeit von Kanaa frustriert?" durch allgemeine kontextuelle Informationen, beispielsweise wenn die Äußerung Teil eines längeren Textes ist

durch Angaben, die der Äußerung selbst zu entnehmen sind.

Sehr often spielen alle drei Arten von Information zusammen, um die Topiksituation zu identifizieren.

\subsection{Die finite Grundstruktur}

Die Überlegungen der vorigen Abschnitte legen demnach folgendes Bild nahe. Wenn ein Satz, zu irgendeiner Gelegenheit geäußert, eine illokutive Funktion erfüllen soll, beispielsweise die, eine Assertion zu machen, dann muß er drei Bestandteile aufweisen:

A. Eine TOPIKKOMPONEnTE. Dies sind all jene Angaben, die dazu beitragen, die Topiksituation zu identifiziern. Dazu gehört zumindest die Topikzeit, in vielen Fällen auch die Angabe einer "Topikwelt" (redet man über die reale oder eine fiktive Welt), ein "Topikort" und eine “Topikentität”. Letztere ist oft durch das grammatische Subjekt ausgedrückt.

B. Eine nicht-finite SATZBASIS. Im einfachsten Fall ist dies ein nichtfinites lexikalisches Verb mit den obligatorischen Ergänzungen, so wie sie von der Rektion des Verbs verlangt werden. ${ }^{9}$

C. Eine BINDEKOMPONENTE. Das ist jener Teil, der Topiksituation und Satzbasis aufeinander bezieht, z.B. indem er markiert, daß letztere für erstere gilt. Dies ist die Funktion der Finitheit.

Die Grundstruktur eines Satzes, der zu einer bestimmten Gelegenheit geäußert wird, ist daher wie folgt:

$$
\text { Äußerung }
$$

$\begin{array}{lll}\text { Topikkomponente } & \text { FIN } & \text { Satzbasis }{ }^{10} \\ \text { Topikzeit -ort -welt -entität } & \text { gibt Relation an } & \begin{array}{l}\text { nichtfinites Verb und Argumente } \\ (+ \text { optionale Elemente })\end{array}\end{array}$

${ }^{9}$ In dieser einfachen Form trifft dies nur zu, wenn die Satzbasis keine lexikalischen Komponenten enthält, die Informationen über die Topiksituation enthalten - beispielsweise in sogenannten "thetischen Sätzen". Ansonsten muß man die Satzbasis aufteilen in Elemente, deren lexikalischer Gehalt die Topikkomponente zu charakterisieren hilft, und Elemente, die dies nicht tun; letzteres ist der "Fokus" oder "Kommentar".

${ }^{10}$ Soweit nicht bestimmte Elemente daraus zur Spezifizierung der Topikkomponente beitragen. Man beachte, daß diese Elemente auch dann zur Satzbasis zählen; in einem gewissen Sinne treten diese Elemente doppelt auf, in charakteristischer Weise beim grammatischen Subjekt in seiner Doppelrolle als Agens (oder sonstiges thematisches Argument) und als "Topikkonstituente" zu beobachten. 
Dieses Schema gibt nur die Bestandteile an. Es besagt nichts darüber, wie sie in einem bestimmten sprachlichen System umgesetzt sind. In den indogermanischen Sprachen werden Finitheit und Angabe der Topikzeit regelhaft durch die Verbflexion miteinander verbunden. Aber das ist nur eine von vielen Möglichkeiten - allerdings eine, die sich eben in der Sprachen der Welt sehr häufig findet und kein Zufall ist. Das Schema sagt auch nicht darüber, wie beispielsweise kenntlich gemacht wird, ob ein bestimmter Teilausdruck zur Topikkomponente gehört oder nicht. Typische Mittel sind Stellung und Intonation. Andere sind expletive Elemente, die die "Topikposition" füllen. Wenn der Satz nicht finit ist, dann gibt es keine Topiksituation, und folglich sind expletive Elemente nicht sinnvoll. Dies erklärt beispielsweise die in $7 \mathrm{~b}$ genannte Lizensierungsbedingung. Im einzelnen sind die hier obwaltenden Regelhaftigkeiten jedoch sehr verwickelt; im vorliegenden Zusammenhang kommt es nur auf die Grundstruktur selbst an.

\subsection{Finite Grundstrukur, Konstruktionsverögen, Kommunikationsvermögen}

Die Grundstruktur, so wie wir sie hier beschrieben haben, beruht letztlich auf den Trivia des Konstruktionsvermögens, wie sie in Abschnitt 3.1 skizziert wurden. Diese Trivia haben sich in den Sprachen der Welt zu einem komplexen, regelhaft zu beobachtenden Organisationsprinzip entfaltet, das für die Form der Kommunikation mit solchen Ausdruckssystemen charakteristisch ist. Dieses Organisationsprinzip beruht auf dem Konstruktionsvermögen, es es hat sich in der Kommunikation entwickelt, und es bestimmt nunmehr die Art und Weise, wie diese Kommunikation funktioniert.

\section{Schluß}

Wie kommt man vom biologisch gegebenen Sprachvermögen $\mathrm{zu}$ den einzelnen Ausdruckssystemen, die dieses Sprachvermögen gebildet hat und deren es sich in der Kommunikation bedient? Hier gibt es zwei grundsätzliche Möglichkeiten, Sprachschöpfung und Spracherwerbs. In beiden Fällen kommen verschiedene Komponenten des Sprachvermögens zum Tragen, die wir als Konstruktionsvermögen K1, als Kopiervermögen K2 und Kommunikationsvermögen K3 bezeichnet haben. Bei der Sprachschöpfung ist K1 bestimmend, K2 jedoch auch beteiligt; die Sprachschöpfung ist ein kollektiver Prozeß, der wechselseitige Nachahmung erfordert. Beim Spracherwerb ist das Verhältnis umgekehrt: es muß in erster Linie Regelhaftigkeiten kopiert werden, ohne daß deshalb ein Mitwirken von K1 ausgeschlossen wäre. In beiden Fällen spielt jedoch auch K3 eine Schlüsselrolle. Es ist dieses Vermögen, das über die Trivia von K1 hinaus die Ausbildung komplexer Teilsysteme ermöglicht, wie sie für natürliche Sprachen charakteristisch sind und die diese so unendlich weit über jede andere Form der Kommunikation erheben.

\section{Literatur}

Anderson, Stephen, Lightfoot, David (2002): The language organ. Cambridge: Cambridge University Press.

Blom, Elma (2001): From root infinitive to finite sentence. Phil. Diss. Utrecht.

Chomsky, Noam (1981): Lectures on Government and Binding. Dordrecht: Foris.

Chomsky, Noam (2002): On Nature and Language. Cambridge: Cambridge University Press. 
Chomsky, Noam, Hauser, Marc D. und, Fitch, Tecumseh (2002): The faculty of language: what is it, what has it, and how did it evolve? In Science 298, S. 1569 - 1579.

Dimroth, Christine, and Lasser, Ingeborg, Hrsg. (2002): Finite options. How L1 and L2 learners cope with the Acquisition of Finiteness. Berlin, New York: Mouton de Gruyter [Sonderheft von Linguistics, 40-4].

Duffield, Nigel (im Druck): Aspects of Vietnamese Clause Structure: separating tense from assertion. Erscheint in Linguistics.

Engels, Friedrich (1869/1962): Der Anteil der Arbeit an der Menschwerdung des Affen. In: MEW 20, $444-445$.

Herder, Johann Gottfried (1784): Ideen zur Philosophie der Geschichte der Menschheit. Riga und Leipzig: Hartknoch.

Klein, Wolfgang und Perdue, Clive (1989): The learner's problem of arranging words. In MacWhinney, Brian, und Bates, Elisabeth, Hrsg., The crosslinguistic study of sentence processing. Cambridge: Cambridge University Press, 1989, S. 292-327.

Klein, Wolfgang, und Perdue, Clive (1997): The Basic Variety, or: Couldn't natural languages be much simpler? In: Second Language Research 13, 301-347.

Longa, Victor M. und Lorenzo, G. (imDruck): What about a (really) minimalist theory of langauge acquisition? Erscheint in Linguistics 2006.

Schumann, John, Favareau, Donald, Goodwin, Charles, Lee, Namhee, Mikesell, Lisa, Tao, Yonghin, Veronique, Daniel, und Wray, Alison (im Druck): Language Evolution: What evolved? Erscheint in Language Learning.

von Stechow, Arnim, und Sternefeld, Wolfgang (1988): Bausteine syntaktischen Wissens. Opladen: Westdeutscher Verlag.

Tallerman, Maggie, Hrsg. (205): Langauge Origins. Respectives on Evolution. Oxford: Oxford University Press.

Ward, Sean, und Trabant, Jürgen (2001): The origins of language. Berlin: Mouton.

Wunderlich, Dieter (2002): Major steps in the evolution of language. Vortrag auf der Sommerschule der DFfS und der LSA, Düsseldorf, Juli 2002 (erhältlich unter http:// ser.philfak.uni-duesseldorf.de/ $\sim$ wdl/lit-them.htm). 